\title{
Las competencias digitales y el uso de las tecnologías sociales: el valor agregado en la comercialización en el sector calzado en Bucaramanga
}

Digital skills and use of social technologies:

value added in marketing in the footwear sector in Bucaramanga

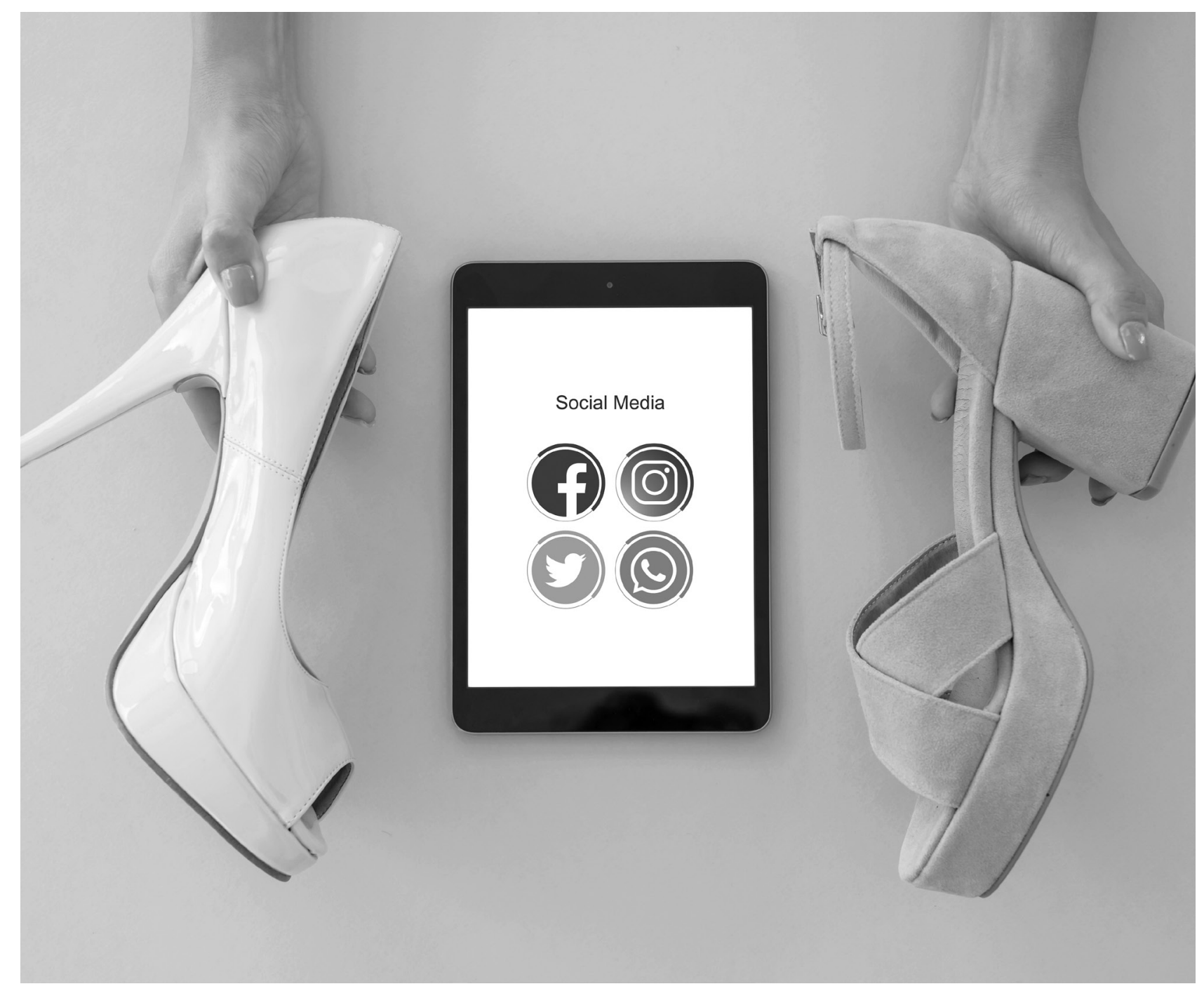




\title{
Las competencias digitales y el uso de las tecnologías sociales: el valor agregado en la comercialización en el sector calzado en Bucaramanga ${ }^{1}$
}

\section{Digital skills and use of social technologies: value added in marketing in the footwear sector in Bucaramanga}

\author{
Alba Patricia Guzmán Duque ${ }^{2}$, Cristian David Gutiérrez Rojas ${ }^{3}$
}

Artículo recibido en octubre de 2016; artículo aceptado en septiembre de 2017.

Este artículo puede compartirse bajo la Licencia Creative Commons Atribución-No Comercial-Compartir lgual 2.0 Genérica

y se referencia usando el siguiente formato: Guzmán, A. \& Gutiérrez, C. (2018). Las competencias digitales y el uso

de las tecnologías sociales: el valor agregado en la comercialización en el sector calzado en Bucaramanga.

I+D Revista de Investigaciones, 11(1), 17-26. DOI: https://doi.org/10.33304/revinv.v11n1-2018002

\begin{abstract}
Resumen
Las condiciones actuales para el desarrollo de la comercialización en las empresas requieren la apropiación de las Tecnologías de la Información y las Comunicaciones [TIC] para generar ventaja competitiva en los sectores de la economía. En este sentido, se necesita el desarrollo de competencias digitales -entendidas como el conjunto de conocimientos, destrezas y actitudes-en los trabajadores de las empresas, para el proceso de actividades relacionadas con el dominio de las TIC y la implementación de las tecnologías sociales en las organizaciones, a fin de mejorar la comercialización de los productos a través de la web. En el presente artículo se plantea cómo las empresas del sector calzado de Bucaramanga, a partir de la capacitación del capital humano en competencias digitales, -búsqueda, procesamiento, comunicación, creación de contenido, trabajo en equipo y difusión por medios tecnológicos- pueden mejorar sus canales de comercialización y lograr el reconocimiento de marca. Se evidencia que las empresas requieren prepararse para incursionar en el mercado considerando la cadena de valor basada en las tecnologías sociales, y que los trabajadores necesitan capacitarse en estos temas para desempeñar sus funciones. La metodología utilizada para desarrollar esta investigación es cualitativa, basada en la revisión de la literatura científica.
\end{abstract}

Palabras clave: competencias digitales, redes sociales, sector calzado, cadena de valor, TIC.

\section{Abstract}

The current conditions for the development of commercialization in companies require the appropriation of Information

1. Artículo científico de enfoque cualitativo, resultado de un proyecto de investigación en curso llamado “Plan de capacitación en competencias digitales para el fortalecimiento del e-commerce en el sector calzado de Bucaramanga", desarrollado en el grupo de investigación Genio, fue financiado por la Universidad Autónoma de Bucaramanga de la ciudad de Bucaramanga (Colombia), Dirección: Avenida 42 n. ${ }^{\circ} 48$ - 11, Bucaramanga - Colombia, PBX (57) (7) 643 6111/643 6261. Fecha de inicio: 1 de julio de 2015. Fecha de terminación: 30 de junio de 2017.

2. Administradora de Empresas, Universidad Nacional de Colombia, 2005. Doctorado Universidad Politécnica de Valencia, doctora en Integración de las TI en las organizaciones, 2013- Docente - investigadora del grupo Genio, Programa Administración de Empresas Modalidad Virtual, Universidad Autónoma de Bucaramanga, Bucaramanga (Colombia), ORCID ID: https://orcid.org/0000-0003-1704-6884. Correo electrónico: aguzman201@unab.edu.co.

3. Administrador de Empresas, Universidad Autónoma de Bucaramanga, 2015. Especialista en Gestión Estratégica de Mercadeo de la Universidad Autónoma de Bucaramanga, 2015 - Joven investigador del grupo Genio, Programa de Negocios Internacionales de la Universidad Autónoma de Bucaramanga (Colombia), ORCID ID: https://orcid.org/0000-0003-3117-7426. Correo electrónico: cgutierrez79@unab. edu.co. 
Alba Patricia Guzmán Duque, Cristian David Gutiérrez Rojas Las competencias digitales y el uso de las tecnologías sociales: el valor agregado en la comercialización en el sector calzado en Bucaramanga

and Communication Technologies (ICT) to generate competitive advantage in the different sectors of the economy. In this sense, it requires the development of digital skills -a set of knowledge, skills and attitudes- in the workers of companies, for the process of activities related to the domain of ICT and the implementation of social technologies in organizations, to improve the commercialization of the products through the web. In this article, the authors explain how companies in the footwear sector of Bucaramanga, based on the training of human capital in digital skills-search, processing, communication, content creation, teamwork and dissemination through technological means- can improve their channels of marketing and achieve brand recognition. It is evident that companies need to prepare to enter the market considering the value chain based on social technologies, and that workers need to be trained in these issues to perform their functions. The methodology used to develop this research is based on qualitative review of the scientific literature.

Keywords: digital skills, social network sites, footwear sector, value chain, ICT.

\section{Introducción}

La tecnología renueva radicalmente la manera como las compañías desarrollan las actividades en sus modelos de negocio puesto que su adopción e implementación ya no es cuestión de elección, sino de necesidad (Coto, 2012). Las Tecnologías de la Información y las Comunicaciones [TIC] han promovido el desarrollo de los países, generando cambios en la forma de actuar de las naciones, influenciando los sectores políticos, económicos y sociales en su búsqueda de ser más competitivos para permanecer en un modelo de mercado globalizado y de permanente cambio (Blandón, 2015; Arras, Torres \& García, 2011). El alcance de las tecnologías sociales muestra un cambio en la forma de vida de la sociedad y en la manera de comunicarse, generando nuevos patrones de consumo, donde las redes sociales se convierten en la herramienta idónea para gestionar los lazos entre la marca y el consumidor estableciendo relaciones en doble vía (Weigend, Dias \& Chow, 2012). Por ejemplo, las grandes economías del mundo como Suiza, Singapur o Estados Unidos cuentan con un mercado laboral competitivo y preparado en comparación con el recurso humano de países en desarrollo como es el caso de Colombia (World Economic Forum [WEF], 2016).

Sin embargo, existen diferentes problemas que implican la necesidad de adoptar las TIC cuando las empresas están buscando contratar personal que esté capacitado para enfrentar el mundo laboral (Conchado, Carot \& Bas, 2015) y que se desempeñe con el uso de las tecnologías. De hecho, la penetración de Internet en Colombia se ha incrementado en los últimos años debido a que ha atravesado un proceso de apertura para la globalización y la eliminación de las barreras de todo tipo, porque permite el intercambio de conocimiento, dando paso al desarrollo y al incremento del uso de las herramientas tecnológicas para navegar por Internet. En Colombia, la penetración de Internet es del $58,6 \%$, lo que se traduce en aproximadamente 28 millones de usuarios, y ha crecido desde el año 2000, cuando eran solo 878000 usuarios que representaban el $2,1 \%$ de la población total del país
(Internet World Stats, 2016). Interactive Advertising Bureau $(I A B, 2015)$ menciona que las tecnologías sociales tienen una penetración del $96,0 \%$ en los usuarios que interactúan permitiendo que herramientas de la web como las redes sociales se conviertan en un instrumento masivo para llegar a diferentes niveles de usuarios de manera ágil y directa (Guzmán \& Del Moral, 2014).

De otro lado, el mercado está en un cambio constante y la Internet, por su facilidad de conectividad, implica que su utilización crezca diariamente en el mundo; este factor obliga a los empresarios a buscar la adopción de la tecnología para ser competitivos frente a los grandes competidores internacionales que se encuentran en el país (Jones, Alderete \& Motta, 2014). Por lo anterior, es necesario que los trabajadores cuenten con las habilidades y las competencias que las empresas requieren para desempeñarse en los cargos de manera eficiente; de hecho, en el sector real se está demandando capital humano capacitado, que posea habilidades para el manejo de las TIC en el desarrollo de las funciones profesionales (Infante, Infante, Martínez \& García, 2016).

Por otra parte, el sector calzado de Bucaramanga es uno de los más importantes y dinamizadores de la región porque genera más de 4000 empleos directos e indirectos en Santander (Colombia). Sin embargo, el sector no es competitivo porque el $80,0 \%$ de las empresas son micro y pequeñas empresas que han surgido de manera empírica, y que no utilizan Internet como medio de comercialización debido a dos factores: la baja capacitación del personal y la entrada de calzado importado con precios por debajo de la producción nacional desde diferentes países, como China. Por este motivo, los empresarios del calzado requieren utilizar las TIC de manera estratégica para que empiecen a comercializar sus productos a través de plataformas tecnológicas, y usar la tecnología social (Guzmán, 2015). Lo anterior permite afirmar que el sector requiere de atención especializada y necesita de capital humano con las competencias demandadas 
por los empresarios en el dominio de las tecnologías sociales, como herramientas que facilitan su desarrollo y el crecimiento (Torres-Coronas \& Vidal-Blasco, 2015).

En este artículo se evidencia cuál es el valor agregado que aportan las tecnologías sociales en los procesos de comercialización de las empresas del sector calzado en Bucaramanga, y se presenta la necesidad que tienen en las empresas de crear perfiles basados en las competencias digitales que requieren los trabajadores del sector para desempeñarse en sus puestos de trabajo relacionados con la comercialización de los productos a través de Internet. El artículo es producto de un proyecto de investigación (en curso), y está desarrollado bajo la metodología de revisión de artículos científicos.

\section{Referente teórico}

\section{Las competencias}

Las organizaciones requieren que su personal cuente con las competencias que evidencien el conjunto de habilidades de sus trabajadores en el desarrollo de una labor específica (Martin \& Omrani, 2015). El concepto de competencia contempla los factores de conocimientos, actitudes, valores y habilidades para ejercer las funciones que se requieren para el desempeño del puesto de trabajo en el ámbito laboral (Martínez, Hernández \& Gómora, 2016; Conchado, Carot \& Bas, 2015).

Las competencias pueden clasificarse según MuñozCantero, Rebollo y Espiñeira (2014) en: genéricas, que van alineadas con las habilidades generales que requieren los trabajadores para desarrollar funciones básicas en el área laboral -aprender a aprender, solucionar los problemas, trabajo en equipo, facilidad comunicativa, entre otras-; $y$ las específicas, que se orientan a evidenciar la capacidad de aplicar las competencias genéricas en un ámbito específico, facilitando la adquisición y generación de conocimientos para el profesional, -las desarrolladas en física, música, idioma extranjero, deporte, el manejo de las TIC, etcétera-.

\section{Competencias digitales}

La nueva era digital invita a las personas a desarrollar habilidades y competencias relacionadas con la tecnología (Vargas-D'Uniam, wChumpitaz-Campos, Suárez-Díaz \& Badia, 2014). Adell (2006) indica que las competencias digitales son el conjunto de competencias para utilizar de manera estratégica las Tecnologías de la Información y las Comunicaciones con el fin de facilitar los procesos de aprendizaje, considerando la optimización de los recursos. Sin embargo, Torres-Coronas y Vidal-Blasco
(2015) afirman que es imprescindible definir el perfil de las competencias genéricas de un trabajador, considerando el análisis del contenido de las ofertas laborales para los empleados, siendo este un aspecto importante para determinar las competencias que están demandando las empresas del sector real y el empalme entre la academia y el mercado (Barhem, Salih \& Darwish, 2008). Para definir las competencias relacionadas con las TIC o competencias digitales o e-competencias o competencias tecnológicas, Torres-Coronas y Vidal-Blasco (2015) realizaron un estudio para determinar el perfil e-competencial de los trabajadores (Ver Tabla 1). Para ello, los autores segmentaron las ofertas laborales en dos áreas del conocimiento, ciencias sociales e ingenierías, determinando las variables afines con las exigencias de los mercados según el área del conocimiento y las funciones a realizar (Delgado \& Trejos, 2016).

\section{Tabla 1}

Competencias digitales de los trabajadores para la realización de sus funciones

\begin{tabular}{|c|c|}
\hline Competencia digital & Variables \\
\hline $\begin{array}{c}\text { Competencias para el } \\
\text { uso de aplicaciones } \\
\text { clave }\end{array}$ & $\begin{array}{l}\text { Nivel competencial en el uso de las } \\
\text { hojas de cálculo (Excel). Nivel } \\
\text { competencial en el uso de } \\
\text { procesadores de texto (Word). } \\
\text { Capacidad de comunicar ideas a } \\
\text { través de presentaciones } \\
\text { (PowerPoint). }\end{array}$ \\
\hline $\begin{array}{l}\text { Fundamentos de } \\
\text { ordenador }\end{array}$ & $\begin{array}{c}\text { Hardware y periféricos. } \\
\text { Sistemas operativos (software). } \\
\text { Comunicación electrónica y } \\
\text { colaboración en línea. } \\
\text { Uso de Internet para la búsqueda y } \\
\text { análisis de información. }\end{array}$ \\
\hline Vivir en red & $\begin{array}{l}\text { Nivel competencial para el uso de } \\
\text { las redes sociales. } \\
\text { Temas legales y de seguridad } \\
\text { relacionadas con internet. }\end{array}$ \\
\hline $\begin{array}{c}\text { Adecuación al mercado } \\
\text { laboral }\end{array}$ & $\begin{array}{l}\text { Nivel de adecuación global } \\
\text { percibido en relación con las } \\
\text { competencias digitales. }\end{array}$ \\
\hline
\end{tabular}

Nota: Competencias digitales según las funciones a realizar, establecidas como herramienta para determinar el perfil competencial del recurso humano de acuerdo con las necesidades de los empresarios. Fuente: Tomado de Torres-Coronas y Vidal-Blasco (2015).

Se observa que las competencias digitales que exigen las empresas se orientan hacia el uso de las TIC debido al beneficio percibido a través del desempeño de las funciones laborales. 
Alba Patricia Guzmán Duque, Cristian David Gutiérrez Rojas Las competencias digitales y el uso de las tecnologías sociales: el valor agregado en la comercialización en el sector calzado en Bucaramanga

De otro lado, el desarrollo de estas competencias digitales muestra un profesional competente considerado como aquel que realiza eficientemente aquellas funciones que debe desarrollar (Arras, Torres \& García, 2011). Además, estos autores señalan que los trabajadores evidencian falencias en cuanto al manejo de las tecnologías de la información, impidiendo la implementación de las TIC en los procesos de las organizaciones.

Suárez, Almerich, Gargallo y Aliaga (2010) afirman que las e-competencias están divididas en seis áreas para desarrollar las habilidades en el uso de las Tecnologías de la Información y las Comunicaciones para su implementación en sus funciones laborales. El uso y manejo de la tecnología, que significa estar contextualizado y tener una disposición amigable y receptiva con el manejo de las TIC; el uso de aplicaciones básicas del ordenador, que son acciones elementales en el uso y la aplicabilidad de las computadoras en los oficios del ámbito laboral; el uso de software de autoedición y presentación, o sea, el manejo de hojas de cálculo, presentaciones, procesadores de texto e Internet; el uso de multimedia, es decir, la competencia para reproducir, cargar y descargar archivos de contenido como videos, imágenes, audios, entre otros; el uso de las tecnologías de la comunicación, supone las habilidades para el manejo de las redes sociales en función de las necesidades de la empresa; y el uso de las tecnologías para el desarrollo de las actividades laborales, se enfoca en aspectos la habilidad para implementar las TIC en los oficios profesionales y laborales para ser más competitivo dentro de las funciones de la empresa.

\section{Tecnologías sociales - Las redes sociales}

El uso de Internet como factor potencial de la comunicación digital ha sido investigado como un elemento que favorece la interacción entre las comunidades a partir del uso de herramientas virtuales (Castañeda, 2010; Coto, 2012; Guzmán \& Del Moral, 2014; Hervás, 2013; Shen \& Bissell, 2013), donde las redes sociales se constituyen en facilitadoras para la comunicación, siendo una necesidad para los trabajadores el desarrollo de sus competencias digitales para el desempeño de las actividades laborales. Precisamente, Castañeda (2010) explica que para finales de los años 90 existían algunas páginas en Internet que podían ser consideradas como redes sociales porque prestaban utilidades particulares como Lycos, red social para la creación de blogs (adquirida por Terra), Geocities, red social de creación de perfiles y contenidos personales (adquirida por Yahoo!), Messenger, red social para mensajería instantánea (adquirida por Skype). Sin embargo, no percibían la importancia de prestar todos los servicios integrados para las organizaciones. Precisamente, a principios del siglo $\mathrm{XXI}$, surgieron nuevas plataformas como Hi5, Fotolog, Bebo, Sónico y MySpace, que ofrecieron un valor agregado: la facilidad de compartir información para comunidades según intereses comunes. En estos sitios se tuvieron importantes volúmenes de tráfico en línea hasta que se creó Facebook, un sitio que en sus inicios alcanzó un rápido crecimiento por su facilidad de uso y la conexión con otros usuarios hasta convertirse en la plataforma que facilita la creación de comunidades virtuales relacionadas con temas que son comunes (Adamic, Lento \& Adar, 2016).

Una red social es una comunidad en línea de usuarios que se encuentran registrados en un sitio web para crear contenido multimedia, hablar, escuchar, reproducir y generar opiniones abiertamente (Hervás, 2013; Shen \& Bissell, 2013). Las características de las redes sociales son: audiencia masiva, porque hay interacción con una gran cantidad de usuarios cada momento en cualquier parte del mundo mediante una conexión a Internet; capacidad de segmentación, puesto que permite la caracterización de los usuarios con intereses comunes; baja percepción de publicidad, en tanto el usuario no reconoce con facilidad la publicidad que se presenta en medio del contenido ya que no se ve expuesto directamente; y la capacidad de creación de contenido, porque permite a los usuarios el consumo de contenido, y basados en sus experiencias y opiniones les permite crear y compartir contenido en la plataforma y con sus grupos de interés (Coto, 2012). Precisamente, Guzmán (2013) las clasificó como generalistas orientadas hacia el público en gen eral (Facebook, Twitter, YouTube) y especializadas dirigidas a un público específico (Linkedln, Xing).

Hoy en día, las redes sociales han ampliado su campo de acción haciendo parte de los procesos de comunicación y de comercialización de los productos a través de los dispositivos con acceso a Internet, beneficiando a los empresarios mediante la reducción de los costos en la planeación y en la ejecución de estrategias de comunicación, la agilización y la automatización de los procesos, la generación de mayor rentabilidad y la segmentación del mercado, favoreciendo la llegada de la empresa al mercado objetivo (Medina \& Castro, 2016).

Precisamente, 9 de cada 10 usuarios de Internet cuentan con al menos un perfil en las redes sociales (The Cocktail Analysis, 2015), siendo Facebook la plataforma más utilizada en el mundo, con una penetración de $96,0 \%$ de los internautas (Actívate, 2016). De otro lado, el portal Alexa.com, una empresa del grupo Amazon.com dedicada a analizar el tráfico en línea de las páginas de Internet, evidencia la popularidad de la web en el mundo, basados en el número de visitas que recibe mensualmente (Alexa, 2016). La Tabla 2 señala la relación entre las redes sociales más utilizadas considerando la penetración de cada una en los internautas y el ranking de visitas según el portal de Alexa.com 
Alba Patricia Guzmán Duque, Cristian David Gutiérrez Rojas

Las competencias digitales y el uso de las tecnologías sociales: el valor agregado en la comercialización en el sector calzado en Bucaramanga

Tabla 2

Penetración y ranking de popularidad de las redes sociales

\begin{tabular}{ccc}
\hline Red social & Penetración en internautas & Ranking de visitas de Alexa (puesto) \\
\hline Facebook & $96,0 \%$ & 3 \\
YouTube & $66,0 \%$ & 2 \\
Twitter & $56,0 \%$ & 8 \\
Google+ & $34,0 \%$ & 14 \\
Linkedln & $31,0 \%$ & 15 \\
Instagram & $26,0 \%$ & 16 \\
\hline
\end{tabular}

Nota:Penetración y ranking de popularidad de las redes sociales. La figura presenta un comparativo entre la penetración que tienen los diferentes usuarios de las redes sociales y la popularidad medida en número de visitas del sitio. Fuente: Autor, a partir de los resultados de The Cocktail Analisys (2015) y del portal Alexa.com (2016).

Se observa que las redes sociales con mayor penetración están posicionadas en los primeros 16 puestos de las páginas más visitadas a nivel mundial, demostrando la relación existente entre Internet y el uso de las redes sociales.

Lo anterior evidencia que Internet y las redes sociales han creado un punto de inflexión en la historia de la comunicación, permitiendo la interacción entre las empresas y los usuarios-consumidores de manera bidireccional. Adicionalmente, las redes sociales hacen parte de la vida cotidiana de los seres humanos, en tanto un $82,0 \%$ de los internautas entre los 18 y 55 años las utilizan (IAB, 2015).

\section{La cadena de valor}

Las organizaciones, para ser competitivas en el mercado, requieren del desarrollo eficiente en cada uno de los procesos, buscando ser diferentes en el mercado y obtener ventaja competitiva. El modelo que describe el proceso para añadir valor agregado al producto desde el proceso de producción hasta la entrega al cliente final y la posventa se denomina cadena de valor (Porter \& Heppelmann, 2015). En este sentido, las organizaciones requieren incluir dentro de sus procesos aspectos que contribuyan a ser escogidos por los clientes para la adquisición de sus productos, considerando que el análisis de la cadena incluye las actividades primarias que se encuentran relacionadas con la transformación de los bienes o de los servicios que serán comercializados y las actividades de soporte que no están directamente relacionadas con la producción pero que son indispensables para agregar valor al producto (Mudambi \& Puck, 2016). Argüello (2015) afirma que este proceso incluye el requerimiento de materias primas, la producción, el procesamiento/transformación, la venta, la comercialización, y la eliminación o reciclaje después del uso, contemplando los factores relacionados con la gestión financiera, los recursos humanos, y la Investigación y Desarrollo (I+D).

La cadena de valor facilita a las organizaciones la mejora en sus procesos para llegar al consumidor final, permitiendo alcanzar la eficiencia en el desarrollo de las actividades primarias y de soporte en la organización, y destacarse sobre otras empresas por ser competitiva (Bravo, 2016). En este sentido, es importante indicar que la tecnología influye directamente en los procesos de desarrollo mediante el aporte de valor a la cadena, beneficiando la productividad de los sectores y dando vía libre a las innovaciones y a la generación de nuevos conocimientos que apunten al cumplimiento de los objetivos de la empresa y a su crecimiento (Ganotakis \& Love, 2012). Lo anterior se evidencia en el proceso de producción y de comercialización a través de diferentes mercados hasta llegar al cliente final, como lo indican Porter y Heppelmann (2015) en su investigación sobre el uso de la tecnología, enfatizando en la facilidad para que los usuarios controlen, a través de estas tecnologías, desde sus coches hasta sus propiedades.

\section{Sector calzado en Bucaramanga}

Colombia es uno de los países líder en la fabricación de calzado después de China, Brasil y México, como lo evidencia Guzmán (2015) en su investigación, donde destacó que la cadena de producción y de distribución para el sector está compuesta por más de 28000 empresas de fabricación, insumo y comercialización del calzado, que contratan más de 180000 trabajadores; esta cifra es equivalente al 7,2\% del empleo en la industria manufacturera. De otro lado, la producción nacional está centralizada en Bogotá, Cali y Bucaramanga; esta última ciudad abarca aproximadamente un $60,0 \%$ del total, con 1424 empresas dedicadas a esta manufactura, 1300 empleos directos y 3900 indirectos en la región (Navas, Díaz \& Rojas, 2014). 
Alba Patricia Guzmán Duque, Cristian David Gutiérrez Rojas Las competencias digitales y el uso de las tecnologías sociales: el valor agregado en la comercialización en el sector calzado en Bucaramanga

Este sector es reconocido en el ámbito nacional por ser uno de los más tradicionales y de los que más aporta a la economía regional (Guzmán, 2015). El Programa de Transformación Productiva [PTP] afirma que el sector está conformado en un $98,0 \%$ por microy pequeñas empresas, indicando que no son competitivas ni están preparadas para enfrentarse al mercado globalizado (Vanguardia Liberal, 2016) debido a que no saben cómo agregar valor a sus procesos, en tanto poseen conocimiento empírico y carecen de un encadenamiento con el sector público y la academia (Ministerio de Comercio, Industria y Turismo, 2013). Ortega (2011) indica que el sector requiere de atención especializada puesto que se encuentra en un proceso de apertura económica y enfrenta un aumento de la competencia.

\section{Metodología}

El enfoque del artículo es cualitativo y está basado en la revisión de artículos científicos relacionados con los temas de competencias digitales, tecnologías sociales, la cadena de valor y el sector calzado en Bucaramanga.

\section{Las tecnologías sociales mecanismo de comunicación con los stakeholders}

El constante avance de la tecnología y de las diferentes formas de comunicación de los usuarios de Internet a través de las herramientas virtuales ha permitido que la conectividad afiance relaciones comerciales entre las empresas y los consumidores. De hecho, las TIC facilitan la comunicación entre las organizaciones y sus stakeholders, siendo este un aspecto relevante para considerar en la fijación de estrategias empresariales que conlleven la mejora de los procesos productivos y comerciales. En este sentido, considerando el alcance de las TIC, el uso de Internet a través de herramientas que agrupan personas o tecnologías sociales es una prioridad para las empresas. Precisamente, las tecnologías sociales se han convertido en una herramienta facilitadora para las comunidades, porque han revolucionado la forma de comunicación de los seres humanos permitiéndoles el acceso a la información y la personalización de productos y servicios. Algunos de los beneficios para las empresas a partir del uso de las TIC son: la reducción de costos, la agilización de procesos, el aumento en la productividad, la realización de transacciones más rentables y el planteamiento de estrategias dirigidas a segmentos específicos. Entre las tecnologías sociales se encuentran las redes sociales, unas poderosas herramientas que fortalecen los lazos entre la marca y el cliente, conectándolos de manera virtual permitiendo a las empresas una conexión directa con los consumidores de sus productos o servicios, facilitando la comprensión de sus gustos y sus parámetros de consumo para ofrecerles productos o servicios casi a la medida. Este aspecto exige de las empresas flexibilidad en sus procesos para adecuarse a los requerimientos del cliente y la inclusión de aspectos relevantes de la cadena de valor para que los productos o servicios ofrecidos sean preferidos por los consumidores y la empresa alcance la competitividad.

Las redes sociales, como herramientas digitales, requieren el desarrollo de habilidades y destrezas que le permitan al empleado utilizarlas de manera eficiente en el desempeño de sus actividades, puesto que son un medio de comunicación efectivo, directo y ágil que está enlazado con los consumidores sin ninguna intermediación, y que requiere ser utilizado por personal competente para hacer efectiva la transacción. Por esta razón las empresas requieren de la implementación de las tecnologías sociales, al tiempo que deben contratar el personal que posea las competencias para el manejo de estas herramientas para el ámbito laboral y comercial, buscando ser más competitivos.

\section{El uso de las tecnologías sociales y la apropiación de las competencias digitales de los trabajadores en el proceso de comercialización de los productos del sector calzado en Bucaramanga como estrategia de valor agregado}

La globalización es una tendencia que permite a las organizaciones, a través del uso de las Tecnologías de la Información y las Comunicaciones, eliminar las barreras físicas para el comercio internacional. El mundo está cambiando y con él las formas tradicionales de los modelos de negocios, que se ven reflejados en los sectores tradicionales de las economías. El sector calzado en Bucaramanga es un potencial dinamizador de la economía santandereana porque genera más de 4000 empleos directos e indirectos en la región. Sin embargo, las compañías de calzado no cuentan con el conocimiento ni la tecnificación suficientes para su desarrollo y crecimiento, razón por la cual se les dificulta llegar al consumidor final a través de estas tecnologías. Sumado a estos aspectos, el sector carece de la coordinación con el gobierno, la academia y el sector real para desarrollar los procesos de comercialización de sus productos; esto implica que los empresarios tengan limitaciones para ser competitivos.

Otro hecho importante para considerar en este problema es la forma en que han surgido los negocios de calzado en Bucaramanga, en tanto que han sido iniciativas empíricas. Este aspecto implica que en el momento de creación de las empresas no se contaba con los conocimientos especializados para plantear un 
Alba Patricia Guzmán Duque, Cristian David Gutiérrez Rojas

Las competencias digitales y el uso de las tecnologías sociales: el valor agregado en la comercialización en el sector calzado en Bucaramanga

modelo productivo que genere valor. Por otro lado, el hecho de que las tendencias del mercado se generan por las exigencias de los consumidores, y el surgimiento de competidores que ofrecen productos a más bajos costos hace que las empresas del sector calzado en Bucaramanga deban replantear sus estrategias de producción y comercialización para llegar al cliente de una forma más directa y ser competitivos en el mercado. En este sentido, la cadena de valor de las empresas de calzado está compuesta por fabricantes, distribuidores, comercializadores y consumidores, los cuales vienen de un modelo de negocio empírico que debe ser reforzado a través de procesos que incluyan las TIC.

Para alcanzar este objetivo, el sector requiere que los gremios, el gobierno y los empresarios le brinden una atención especializada, puesto que los procesos económicos -como la apertura económica y el aumento de la competencia- requieren de la utilización e implementación de las TIC para ser competitivos. Precisamente, como los patrones de consumo se han transformado, las organizaciones se ven obligadas a cambiar el foco de atención del producto y centrarlo en las necesidades del consumidor para atenderlas de manera eficiente. En este escenario, la inclusión de procesos de intercambio de información a través de las TIC facilita la orientación de los esfuerzos estratégicos para llegar al consumidor de manera oportuna.

Sin embargo, las compañías del sector calzado deben considerar otro factor que se asocia con su naturaleza. Las empresas son organizaciones conformadas por un conjunto de trabajadores que realizan sus funciones para aportar al cumplimiento de los objetivos organizacionales. Por lo anterior, el recurso humano se convierte en uno de las más importantes de las áreas funcionales de las empresas, siendo el responsable de ejecutar los procesos para que la empresa sea competitiva. En este sentido, se requiere que los nuevos trabajadores desarrollen las habilidades tecnológicas requeridas por el perfil de la empresa, es decir, que implementen las TIC en sus labores profesionales a través de la fijación de las competencias digitales para enlazar la tecnología con los procesos de la cadena de valor de la compañía. En este escenario, donde las empresas requieren utilizar las tecnologías sociales como un mecanismo para agregar valor a sus procesos y ser competitivas, como ya se ha mencionado, las redes sociales fortalecen las relaciones con los clientes para generar el reconocimiento de marca y mejorar la percepción de la imagen corporativa en los consumidores. Este hecho las convierte en un canal de comunicación que favorece la interacción con los usuarios y que puede transformarse en el elemento clave que le agregue valor al proceso de comercialización de los productos de los empresarios del sector calzado de Bucaramanga.
Todo lo anterior permite concluir que existen dos herramientas importantes que deben considerarse en la cadena de valor de las empresas de calzado por su aporte a la ventaja competitiva y al valor diferencial que ofrecen frente a la competencia: las tecnologías sociales y las competencias digitales. Estas herramientas tecnológicas permiten que el trabajador interactúe a través de contenido multimedia con el consumidor y recibir sus opiniones para la mejora del servicio, así como, que de manera global, la marca llegue a una audiencia masiva a fin de abrirse nuevos mercados, explorar nuevos campos, segmentar los esfuerzos de la compañía y reforzar los lazos con los integrantes de la cadena de valor.

Finalmente, es importante indicar que, tras incluir las tecnologías sociales en su cadena de valor, las empresas del sector calzado de Bucaramanga requieren plantear estrategias de comunicación y comercialización en sus procesos que les permitan ser competitivas en el mercado. En consecuencia, se puede afirmar que las competencias digitales brindan habilidades en el manejo de las TIC a los trabajadores para desempeñarse de manera eficiente en sus funciones, utilizando las redes sociales como canal de comunicación y de comercialización con los usuarios o consumidores, permitiéndoles a las empresas alcanzar la ventaja competitiva.

\section{Referencias}

Actívate. (2016). Curso Actívate, Google España 2016. Interactive Advertising Bureau. Recuperado de https://cursos. formacionactivate.es/marketing-digital/course

Adamic, L., Lento, T. \& Adar, E. (2016). Information evolution in social networks. In Proceedings of the Ninth ACM International Conference on Web Search and Data Mining (pp. 473-482).

Adell, J. (2006). Internet en el aula: las WebQuest. Edutec. Revista Electrónica De Tecnología Educativa, (17) Recuperado de https://doi.org/10.21556/edutec.2004.17.530

Alexa.com. (2016). The top 500 sites on the web. Recuperado de http://www.alexa.com/topsites

Arguello, G. (2015). Uso de las TIC en las PYME del sector industrial del Área Metropolitana de Bucaramanga. I+ $D$ Revista de Investigaciones, 6(2), 125-136.

Arras, A., Torres, C. \& García, A. (2011). Competencias en Tecnologías de Información y Comunicación (TIC) de los estudiantes universitarios. Revista Latina de Comunicación Social, 6(66), 1-26. Recuperado de http://www. revistalatinacs.org/11/art/927_Mexico/06_Arras.html

Barhem,B.,Salih,A.\&Darwish,A.(2008).Thebusinesscurriculum and the future employment market: UAE business leaders' views. Education, Business and Society: Contemporary Middle Eastern Issues, 1(2) 124-137. Recuperado de https:// doi.org/10.1108/17537980810890301 
Alba Patricia Guzmán Duque, Cristian David Gutiérrez Rojas Las competencias digitales y el uso de las tecnologías sociales: el valor agregado en la comercialización en el sector calzado en Bucaramanga

Blandón, A. (2015). Acuerdos regionales de competitividad y 'gobernabilidad' en las cadenas de valor (cv). El caso de la CV cacao-chocolate región nororiental. Revista Mundo Económico y Empresarial, (6), 63-70.

Bravo, V. (2016). Competitividad y costos. Revista Publicando, $3(7), 312-321$.

Castañeda, L. (2010). Aprendizaje con redes sociales. Tejidos educativos para los nuevos entornos. Sevilla: MAD Eduforma.

Conchado, A., Carot, J. \& Bas, M. (2015). Competencies for knowledge management: development and validation of a scale. Journal of Knowledge Management, 19(4), 836-855.

Coto, M. (2012). El plan de marketing digital: blanded marketing como integración de acciones on y offline. Recuperado de https://www.researchgate.net/publication/44107034_El_ plan_de_marketing_digital_Blended_Marketing_como_ integracion_de_acciones_on_y_offline_M_Alonso_Coto_ prol_de_Jose_Maria_Sanabria_Gildo_Seisdedos

Delgado, S. \& Trejos, C. (2016). Análisis organizacional de las Pymes sector comercio de calzado zona centro, Pasto. I+ D Revista de Investigaciones, 7(1), 98-108.

Ganotakis, P. \& Love, J. (2012). The innovation value chain in new technology-based firms: Evidence from the UK. Journal of product innovation management, 29(5), 839-860.

Guzmán, A. (2013). Factores críticos de éxito en el uso de las redes sociales en elámbito universitario: aplicación aTwitter. (Doctoral dissertation): Universitat Politècnica de València).

Guzmán, A. (2015). "E-commerce: Competencias digitales en el sector del calzado en Bucaramanga para aumentar la competitividad utilizando las TIC al servicio del teletrabajo". Sistema de Investigaciones Siunab (pp. 3-5).

Guzmán, A. \&Del Moral, M. (2014).Tendencias de uso de YouTube: optimizandolacomunicaciónestratégicadelasuniversidades iberoamericanas. Observatorio (OBS*), 8(1), 69-94.

IAB (2015). VI Estudio Anual Redes Sociales de LAB Spain. Recuperado dehttp://www.iabspain.net/wpcontent/ uploads/downloads/2015/01/Estudio_Anual_Redes_ Sociales_2015.pdf

Infante, A., Infante, J., Martínez, F. \& García, M. (2016). Las competencias digitales en las grandes empresas del sector empresarial español. (Digital skills in big companies of the Spanish business sector). Tec Empresarial, 10(2), 4149. Recuperado de http:// revistas.tec.ac.cr/index.php/ tec_empresarial/article/view/2654/2383

Internet World Stats. (2016). Colombia, Internet usage indicators, and country profile. Recuperado de http:// www. internetworldstats.com/sa/co.htm

Jones, C., Alderete, M. \& Motta, J. (2014). Adopción del comercio electrónico en micro, pequeñas y medianas empresas comerciales y de servicios de Córdoba, Argentina. Cuadernos de Administración, 29(50), 164-175.

Martin, L. \& Omrani, N. (2015). An assessment of trends in technology use, innovative work practices and employees' attitudes in Europe. Applied Economics, 47(6), 623-638'.

Martínez, M., Hernández, M. \& Gómora, Y. (2016). Modelo de competencias directivas en escenarios globales para las instituciones de educación superior. RIDE Revista iberoamericana para la investigación y el desarrollo educativo, 6(12). 321-333. Recuperado de https://dialnet. unirioja.es/servlet/articulo?codigo $=5415448$

Medina, M. \& Castro, Y. (2016). La adopción del marketing digital en empresas de Aguascalientes. Red Internacional de Investigadores en Competitividad, 9(1), 386-401.

Ministerio de Comercio, Industria y Turismo. (2013). Así avanza la transformación productiva 2013. (Programa deTransformación Productiva). Recuperado dehttps:// www.ptp.com.co/documentos/INFORME\%20 COMPLETO\%20PTP.pdf

Mudambi, R. \& Puck, J. (2016). A Global Value Chain Analysis of the 'Regional Strategy 'Perspective. Journal of Management Studies. 6(53), 1076-1093. Recuperado de http:// onlinelibrary.wiley.com/doi/10.1111/joms.12189/pdf

Muñoz-Cantero, J., Rebollo, N. \& Espiñeira, E. (2014). Percepción de competencias en el EEES: análisis en el Grado de Educación Primaria. Revista Electrónica Interuniversitaria de Formación del Profesorado, 17(3), 123-140. Recuperado de http://dx.doi.org/10.6018/reifop.17.3.204091

Navas, J., Díaz, O. \& Rojas, M. (2014). La competitividad bajo el modelo del diamante de Porter: un reto para la industria del calzado de Bucaramanga. Recuperado de http:// intellectum.unisabana.edu.co/handle/10818/12112

Ortega, W. (2011). Estructura y dinámica competitiva del sector calzado en Bucaramanga. Memorias, 9(15), 55-68.

Porter, M. \& Heppelmann, J. (2015). How smart, connected products are transforming companies. Harvard Business Review, 93(10), 53-71.

Shen, B.\&Bissell, K. (2013).Social media, social me: A contentanalysis of beauty company's use of Facebook in marketing and branding.Journal of Promotion Management, 19(5), 629-651.

Suárez, J, Almerich, G., Gargallo, B. \& Aliaga, F. (2010). Las competencias en TIC del profesorado y su relación con el uso de los recursos tecnológicos. Archivos Analíticos de Políticas Educativas, 18(10), 1-33.

The Cocktail Analysis. (2015). Presentación de resultados Observatorio Redes Sociales VII Ola. Recuperado de http:// tcanalysis.com/blog/posts/vii-observatorioredes-sociales

Torres-Coronas, T. \& Vidal-Blasco, M. (2015). Students and employers perception about the development of digital skills in higher education. Revista deeducación, 367(27), 63-90.

Vanguardia Liberal. (julio, 2016). Colombia no impondrá antidumping a calzado. Recuperado de http://www. vanguardia.com/economia/nacional/366477colombiano-impondra-antidumping-a-calzado

Vargas-D'Uniam, J., Chumpitaz-Campos, L., Suárez-Díaz, G. \& Badia, A. (2014). Relación entre las competencias digitales de docentes de educación básica y el uso educativo de las tecnologías en el aula. Profesorado: Revista de curriculum y formación del profesorado, 18(3), 361-377.

World Economic Forum. (2016). World economic forum annual 
meeting 2016: mastering the fourth industrial revolution. Recuperado de https://www.weforum.org/reports/worldeconomic-forum-annual-meeting-2016mastering-thefourth-industrial-revolution

Weigend, A., Dias, G. \& Chow, A. (2012). Tecnologías sociales. El poder de las conversaciones en red. Recuperado de http:// craig.com.ar/biblioteca/1/Tecnologias $\% 20$ Sociales $\% 20$ \%20El\%20poder\%20de\%20las\%20conversaciones\%20 en\%20red.pdf. 